\title{
On Silicon Group Elements Ejected by Supernovae Type Ia
}

\author{
Soma De ${ }^{1}$, F. X. Timmes ${ }^{1,2}$ Edward F. Brown ${ }^{2,3}$, Alan C. Calder ${ }^{4,5}$, Dean M. Townsley ${ }^{6}$, \\ Themis Athanassiadou ${ }^{7}$, David A. Chamulak ${ }^{8}$, Wendy Hawley ${ }^{9}$, Dennis Jack ${ }^{10}$ \\ somad@asu .edu
}

\begin{abstract}
There is compelling evidence that the peak brightness of a Type Ia supernova is affected by the electron fraction $Y_{\mathrm{e}}$ at the time of the explosion. The electron fraction is set by the aboriginal composition of the white dwarf and the reactions that occur during the pre-explosive convective burning. To date, determining the makeup of the white dwarf progenitor has relied on indirect proxies, such as the average metallicity of the host stellar population. In this paper, we present analytical calculations supporting the idea that the electron fraction of the progenitor systematically influences the nucleosynthesis of silicon group ejecta in Type Ia supernovae. In particular, we suggest the abundances generated in quasi nuclear statistical equilibrium are preserved during the subsequent freezeout. This allows one to potential recovery of $Y_{\mathrm{e}}$ at explosion from the abundances recovered from an observed spectra. We show that measurement of ${ }^{28} \mathrm{Si},{ }^{32} \mathrm{~S},{ }^{40} \mathrm{Ca}$, and ${ }^{54} \mathrm{Fe}$ abundances can be used to construct $Y_{\mathrm{e}}$ in the silicon-rich regions of the supernovae. If these four abundances are determined exactly, they are sufficient to recover $Y_{\mathrm{e}}$ to $6 \%$. This is because these isotopes dominate the composition
\end{abstract}

\footnotetext{
${ }^{1}$ School of Earth and Space Exploration, Arizona State University, Tempe, AZ

${ }^{2}$ Joint Institute for Nuclear Astrophysics

${ }^{3}$ Department of Physics \& Astronomy, Michigan State University, East Lansing, MI

${ }^{4}$ Department of Physics \& Astronomy, Stony Brook University, Stony Brook, NY

${ }^{5}$ Institute for Advanced Computational Science, Stony Brook University, Stony Brook, NY

${ }^{6}$ Department of Physics \& Astronomy, The University of Alabama, Tuscaloosa, AL

${ }^{7}$ Swiss National Supercomputing Centre, Via Trevano 131, 6900 Lugano, Switzerland

${ }^{8}$ Physics Division, Argonne National Laboratory, Argonne, IL

${ }^{9}$ Laboratoire d'Astrophysique de Marseille, Marseille cedex 13, France

${ }^{10}$ Departamento de Astronomía, Universidad de Guanajuato, Apartado Postal 144, 36000, Guanajuato, Mexico
} 
of silicon-rich material and iron-rich material in quasi nuclear statistical equilibrium. Analytical analysis shows that the ${ }^{28} \mathrm{Si}$ abundance is insensitive to $Y_{\mathrm{e}}$, the

${ }^{32} \mathrm{~S}$ abundance has a nearly linear trend with $Y_{\mathrm{e}}$, and the ${ }^{40} \mathrm{Ca}$ abundance has a nearly quadratic trend with $Y_{\mathrm{e}}$. We verify these trends with post-processing of $1 \mathrm{D}$ models and show that these trends are reflected in the model's synthetic spectra.

Subject headings: nuclear reactions, nucleosynthesis, abundances — supernovae: general — white dwarfs

\section{Introduction}

Type Ia supernovae (henceforth SNIa) are thought to be the evolutionary terminus for a class of binary stellar systems (Whelan \& Iben 1973; van den Heuvel et al. 1992; Kahabka \& van den Heuvel 1997; Parthasarathy et al. 2007; Meng \& Yang 2010), the thermonuclear incineration of one or more carbon-oxygen white dwarfs (Branch et al. 1995; Wang \& Han 2012), a primary source of iron in galaxies (Matteucci \& Greggio 1986; Tang \& Wang 2010; Bulbul et al. 2012), accelerators of cosmic rays and sources of kinetic energy in galaxy evolution (Wang 2011; Powell et al. 2011), and useful tools for measuring cosmological parameters (Phillips 1993; Riess et al. 1998; Perlmutter et al. 1999; Kowalski et al. 2008; Wood-Vasey et al. 2008; Hicken et al. 2009; Riess et al. 2011; Conley et al. 2011; Foley \& Kasen 2011; Foley et al. 2011; Sullivan et al. 2011; Suzuki et al. 2012; Silverman et al. 2012a b c).

The peak luminosity of SNIa is set by the radioactive decay chain ${ }^{56} \mathrm{Ni} \rightarrow{ }^{56} \mathrm{Co} \rightarrow$ ${ }^{56} \mathrm{Fe}$ (Arnett 1979; Colgate et al. 1980; Arnett 1982; Arnett et al. 1985), and the observed photometric correlation between the peak luminosity and the timescale over which the light curve decays from its maximum (Phillips 1993) is understood physically as having both the luminosity and opacity being set by the mass of ${ }^{56} \mathrm{Ni}$ synthesized in the explosion Arnett 1982; Pinto \& Eastman 2000; Mazzali \& Podsiadlowski 2006; Kasen \& Woosley 2007). When corrected for the correlation between peak luminosity and light curve decay timescale, the intrinsic dispersion in SNIa distances is $\sim 0.14 \mathrm{mag}$ ( $\leq 7 \%$ in distance, Jha et al. 2007), not all of which can be attributed to statistical error. This correction removes the dispersion that is attributed primarily from the difference in ${ }^{56} \mathrm{Ni}$ mass and other physical effects. For example, the residual dispersion in the Hubble diagram is reduced by excluding those SNIa with high-velocity ejecta (Foley \& Kasen 2011; Foley et al. 2011; Foley 2012). Furthermore, there appears to be a need to correct for the host galaxy (Howell 2011) as some properties of the host stellar population are apparently imprinted on the explosion. Accounting for such systematic effects potentially allows for more accurate determinations of the distance 
modulus from the observed light curve and spectra. This implies identifying and extracting observable physical effects that may create a dispersion between the SNIa light curves.

Over the last decade a number of observational and theoretical studies have sought to uncover such systematic effects from variables other than ${ }^{56} \mathrm{Ni}$, with the aim of making distance measurements more precise and improving our quantitative understanding of the progenitor systems. Examples include the empirical correlations between the spectra and light curve (Barbon et al. 1990; Branch et al. 1993; Nugent et al. 1995; Blondin \& Mezzacappa 2006; Bongard et al. 2006; Branch et al. 2009; Nordin et al. 2011; Blondin et al. 2011; Foley \& Kasen 2011; Foley 2012), the dependence of the peak brightness on the progenitor metallicity (Höflich et al. 1998; Umeda et al. 1999; Timmes et al. 2003; Travaglio et al. 2005; Röpke et al. 2006; Ellis et al. 2008; Gallagher et al. 2008; Piro \& Bildsten 2008; Chamulak et al. 2008; Badenes et al. 2008; Howell et al. 2009; Neill et al. 2009; Townsley et al. 2009; Sullivan et al. 2010; Jackson et al. 2010; Bravo \& Badenes 2011; Foley \& Kirshner 2013), asymmetries in the explosion (Howell 2001; Kasen et al. 2003, 2004; Wang et al. 2008; Kasen et al. 2009; Chamulak et al. 2012), central density and carbon-oxygen ratio (Höflich et al. 1998; Domínguez et al. 2001; Röpke et al. 2006), age of the progenitor (Scannapieco \& Bildsten 2005; Mannucci et al. 2006; Sullivan et al. 2006, 2010; Krueger et al. 2010, 2012), abundance ratios of neutron-rich isotopes to ${ }^{56} \mathrm{Ni}$ (Mazzali \& Podsiadlowski 2006), and the opacity of the overlying material (Mazzali et al. 2001; Kasen \& Woosley 2007). A consensus is still lacking, however, on the progenitor systems as well as on how differences in initial conditions create variances in the observed properties of SNIa.

The composition of the white dwarf should have an effect on the nucleosynthesis during the explosion and thus on the isotopic abundances of the final composition. For example, most of a main-sequence star's initial metallicity comes from the CNO and ${ }^{56}$ Fe nuclei inherited from its ambient interstellar medium. The slowest step in the hydrogen burning CNO cycle is proton capture onto ${ }^{14} \mathrm{~N}$. Consequently, all catalyst nuclei are converted to ${ }^{14} \mathrm{~N}$ when hydrogen core burning on the main sequence is completed. During helium core burning the reaction sequence ${ }^{14} \mathrm{~N}(\alpha, \gamma){ }^{18} \mathrm{~F}\left(\beta^{+} \nu_{e}\right){ }^{18} \mathrm{O}(\alpha, \gamma){ }^{22} \mathrm{Ne}$ converts most of the ${ }^{14} \mathrm{~N}$ into ${ }^{22} \mathrm{Ne}$. From this point forward, stars have a net positive neutron excess $\eta$, defined as $\eta=1-2\langle Z\rangle /\langle A\rangle=1-2 Y_{\mathrm{e}}$ where $\langle Z\rangle$ is the mean atomic number, $\langle A\rangle$ is the mean nucleon number, and $Y_{\mathrm{e}}$ is the electron fraction. Additional burning stages will be driven towards producing more neutron-rich elements at the expense of other elements.

For example, this net neutron excess increases the production of neutron-rich isotopes such as ${ }^{54} \mathrm{Fe}$ and ${ }^{58} \mathrm{Ni}$ instead of radioactive ${ }^{56} \mathrm{Ni}$ in the regions of the white dwarf that produce most of the iron group isotopes during the explosion. This leads to a linear correlation between the birth metallicity and the peak brightness (Timmes et al. 2003; Röpke et al. 2006; 
Foley \& Kirshner 2013). However, the range of birth metallicities $Z_{\text {birth }}$ is not large enough to account for the full diversity of SNIa peak luminosity (Gallagher et al. 2005, 2008; Howell et al.|2009). This has encouraged exploration of other factors that may impact neutronization prior to the explosion. Some explorations have focused on the $\sim 1000 \mathrm{yr}$ long convective simmering of the white dwarf prior to explosion. The convective region is driven by the ${ }^{12} \mathrm{C}+{ }^{12} \mathrm{C}$ reaction and extends outward in mass from from the core to $\approx 1.2 M_{\odot}($ Piro \& Chang 2008). During this simmering phase the reaction sequence ${ }^{12} \mathrm{C}(\mathrm{p}, \gamma)^{13} \mathrm{~N}\left(e^{-}, \nu_{e}\right)^{13} \mathrm{C}$ increases the neutron excess by an amount that depends on the total mass of carbon burned prior to the explosion (Piro \& Bildsten 2008; Chamulak et al. 2008). Such studies have established the existence of a "floor" level of neutronization that is larger than the neutronization due to the birth metallicity when $Z_{\text {birth }} \lesssim 2 / 3 Z_{\odot}$, where $Z$ is the metallicity. Thus, simmering may mask correlations between SNIa properties and the birth metallicity. For the purpose of this paper, however, what matters is that the white dwarf has a well defined $Y_{\mathrm{e}}$ when it explodes, and not how the white dwarf achieved that $Y_{\mathrm{e}}$ distribution.

On the other hand, observational SNIa surveys exploring the impact of potential metallicity effects invariably use the metallicity of the host galaxy as a proxy for the metallicity of the progenitor white dwarf (Ellis et al. 2008; Gallagher et al. 2008; Howell et al. 2009; Neill et al. 2009; Sullivan et al. 2010). It is well established, however, that there is a relatively large scatter in stellar iron to hydrogen ratios, $\Delta[\mathrm{Fe} / \mathrm{H}] \sim 0.5 \mathrm{dex}$, at any given age for stars in the Milky Way (Twarog 1980; Edvardsson et al. 1993; Chen et al. 2000; Feltzing et al. 2001; Rolleston et al. 2000; Pedicelli et al. 2009). For example, Feltzing et al. (2001) constructed an age-metallicity diagram for 5828 dwarf and sub-dwarf stars from the Hipparcos Catalog using evolutionary tracks to derive ages and Strömgren photometry to derive metallicities. They conclude that the age-metallicity diagram is well-populated at all ages, that old but metal-rich stars exist, and that the scatter in metallicity at any given age is larger than the observational uncertainties. Alternatively, by following the chemical evolution of homogeneous galaxy models with the evolution of the supernova rates in order to evaluate the metallicity distribution function, Bravo \& Badenes (2011) find the mean metallicity of SNIa and the metallicity of the host galaxy are tightly correlated when both metallicities are measured as the $\mathrm{CNO}$ abundance.

If the composition of the white dwarf has an observable effect on the ${ }^{56} \mathrm{Ni}$ production and thus the SNIa light curve, it could have an effect on other elements as well. In this paper we present a new direct method to measure the electron fraction $Y_{\mathrm{e}}$ in the silicon-rich regions for individual SNIa by using observed abundances of $\mathrm{Si}, \mathrm{S}, \mathrm{Ca}$, and Fe. Our method follows from the twin facts that ${ }^{28} \mathrm{Si},{ }^{32} \mathrm{~S},{ }^{40} \mathrm{Ca}$ and ${ }^{54} \mathrm{Fe}$ are produced in a quasi nuclear statistical equilibrium (henceforth QNSE) environment, and that the abundance levels achieved during QNSE do not change during the subsequent freezeout as the SNIa expands. Thus, the 
QNSE abundance levels of these elements are recorded in the spectra. Working in reverse, from the observed abundances we can apply the QNSE relations to determine the QNSE abundances and thus determine a reasonably accurate measure of $Y_{\mathrm{e}}$ in the silicon group producing regions. This method is independent of any SNIa explosion model and assumes only that the isotopes are synthesized in a QNSE state. Our primary motivation behind constraining $Y_{\mathrm{e}}$ is to reduce the residual dispersion in the Hubble diagram by correcting for a potentially measurable systematic effect. Our secondary aim is to provide rigorous nucleosynthesis constraints that can guide the modeling of SNIa synthetic spectra.

Our paper is organized as follows. In \$2 we establish the QNSE equations that connect the abundances to $Y_{\mathrm{e}}$ and present a method for constructing the electron fraction from the ${ }^{28} \mathrm{Si},{ }^{32} \mathrm{~S},{ }^{40} \mathrm{Ca}$ and ${ }^{54} \mathrm{Fe}$ abundances. In $\$ 3$ we show the trends predicted by our QNSE based relations are present in the nucleosynthesis and spectra of common 1D SNIa models. Finally, in Section 5 we discuss the implications of our results.

\section{Constructing $Y_{\mathrm{e}}$ from measured abundances}

In this section we develop a framework based on equilibrium thermodynamics and the conservation laws that allows construction of the electron fraction $Y_{\mathrm{e}}$ from the major abundances in QNSE silicon-rich material. We then conclude this section by showing the principle functional dependencies of the silicon group (henceforth $\mathrm{SiG}$ ) and iron group (henceforth FeG) on $Y_{\mathrm{e}}$.

\subsection{Basic framework}

We first select a system consisting of the major $\mathrm{SiG}$ and FeG elements to trace out the most useful equations connecting the individual abundances and their relationship to $Y_{\mathrm{e}}$. We choose ${ }^{28} \mathrm{Si},{ }^{32} \mathrm{~S}$, and ${ }^{40} \mathrm{Ca}$ from the $\mathrm{SiG}$ isotopes, and ${ }^{58} \mathrm{Ni}$ and ${ }^{54} \mathrm{Fe}$ from the FeG isotopes. As Figure 1 suggests, these are the dominant isotopes under QNSE conditions. Conservation of mass and charge can therefore be expressed as

$$
\begin{aligned}
Y_{\mathrm{n}}+Y_{\mathrm{p}}+28 Y_{28 \mathrm{Si}}+32 Y_{32 \mathrm{~S}}+40 Y_{40 \mathrm{Ca}}+54 Y_{54 \mathrm{Fe}}+58 Y_{58 \mathrm{Ni}} & =1 \\
Y_{\mathrm{p}}+14 Y_{28 \mathrm{Si}}+16 Y_{32 \mathrm{~S}}+20 Y_{40 \mathrm{Ca}}+26 Y_{54 \mathrm{Fe}}+28 Y_{58 \mathrm{Ni}} & =Y_{\mathrm{e}}
\end{aligned}
$$

From minimization of the Helmholtz free energy there follows the fundamental QNSE relations (Bodansky et al. 1968; Hix \& Thielemann 1996; Meyer et al. 1998; Iliadis 2007)

$$
\frac{Y_{A, Z}}{Y_{A^{\prime}, Z^{\prime}}}=f(\rho, T) Y_{\mathrm{p}}^{Z-Z^{\prime}} Y_{\mathrm{n}}^{A-A^{\prime}-\left(Z-Z^{\prime}\right)}
$$




$$
\begin{aligned}
f(\rho, T) & =\frac{G_{A, Z}}{G_{A^{\prime}, Z^{\prime}}}\left(\frac{\rho N_{\mathrm{A}}}{\theta}\right)^{A-A^{\prime}} \exp \left(\frac{B-B^{\prime}}{k_{\mathrm{B}} T}\right), \\
\theta & =\left(\frac{m_{\mathrm{u}} k_{\mathrm{B}} T}{2 \pi \hbar^{2}}\right)^{\frac{3}{2}} .
\end{aligned}
$$

Here $T$ is the temperature, $\rho$ is the baryonic mass density, $G_{A, Z}$ is the temperature-dependent partition function, $B$ is the nuclear binding energy, $N_{\mathrm{A}}$ is the Avogadro constant, $k_{\mathrm{B}}$ is the Boltzmann constant, and $m_{\mathrm{u}}$ is the atomic mass unit. The molar abundances are the local abundances that correspond to a region of the star associated with a specific $\rho$ and $T$. The nuclei are treated as an ideal gas and we ignore screening corrections, both of which are justifiable assumptions under the thermodynamic conditions of interest. Specification of $T$, $\rho, Y_{\mathrm{e}}$, the aggregate molar abundance of the $\mathrm{SiG}$ isotopes $Y_{\mathrm{SiG}}$, and the aggregate molar abundance of the FeG isotopes $Y_{\mathrm{FeG}}$ is sufficient to solve for all the abundances in a twocluster QNSE environment. At a given $\rho$ and $T$, we use Equation (3) to write $Y_{32 \mathrm{~S}}$ and $Y_{40 \mathrm{Ca}}$ in terms of $Y_{28 \mathrm{Si}}, Y_{\mathrm{p}}$, and $Y_{\mathrm{n}}$. Similarly $Y_{58 \mathrm{Ni}}$ is written in terms of $Y_{54 \mathrm{Fe}}, Y_{\mathrm{p}}$, and $Y_{\mathrm{n}}$. This leaves us with four unknowns, $Y_{\mathrm{p}}, Y_{\mathrm{n}}, Y_{28 \mathrm{Si}}$, and $Y_{54 \mathrm{Fe}}$, and, for a known $Y_{\mathrm{e}}$, four constraints: Equations (1) and (2), and the sums $Y_{\mathrm{SiG}}=Y_{28 \mathrm{Si}}+Y_{32 \mathrm{~S}}+Y_{40 \mathrm{Ca}}$ and $Y_{\mathrm{FeG}}=Y_{54 \mathrm{Fe}}+Y_{58 \mathrm{Ni}}$, which are both specified externally to the solution of the QNSE.

Measurement of four quantities $Y_{28 \mathrm{Si}}, Y_{32 \mathrm{~S}} / Y_{28 \mathrm{Si}}, Y_{40 \mathrm{Ca}} / Y_{32 \mathrm{~S}}$, and $Y_{54 \mathrm{Fe}} / Y_{28 \mathrm{Si}}$ is an equally sufficient basis from which to solve for all the abundances in the silicon-rich region of SNIa. For our choice of isotopes, Equation (3) leads to

$$
\begin{aligned}
\frac{Y_{28 \mathrm{Si}}}{Y_{32 \mathrm{~S}}} \approx\left(\frac{\rho N_{\mathrm{A}}}{\theta}\right)^{-4} Y_{\mathrm{p}}^{-2} Y_{\mathrm{n}}^{-2} \exp \left(\frac{B_{28 \mathrm{Si}}-B_{32 \mathrm{~S}}}{k_{\mathrm{B}} T}\right) \\
\frac{Y_{54 \mathrm{Fe}}}{Y_{58 \mathrm{Ni}}} \approx\left(\frac{\rho N_{\mathrm{A}}}{\theta}\right)^{-4} Y_{\mathrm{p}}^{-2} Y_{\mathrm{n}}^{-2} \exp \left(\frac{B_{54 \mathrm{Fe}}-B_{58 \mathrm{Ni}}}{k_{\mathrm{B}} T}\right) \\
\frac{Y_{32 \mathrm{~S}}}{Y_{40 \mathrm{Ca}}} \approx\left(\frac{\rho N_{\mathrm{A}}}{\theta}\right)^{-8} Y_{\mathrm{p}}^{-4} Y_{\mathrm{n}}^{-4} \exp \left(\frac{B_{32 \mathrm{~S}}-B_{40 \mathrm{Ca}}}{k_{\mathrm{B}} T}\right) .
\end{aligned}
$$

Here we assume all ratios of nuclear partition functions are unity. This is justifiable, as at typical QNSE temperatures the nuclei are mostly in their ground state, and all of these nuclei have zero spin.

Using Equations (6) and (8), consider the local SiG element ratio

$$
\begin{aligned}
\Phi(T) & =\frac{Y_{28 \mathrm{Si}}}{Y_{32 \mathrm{~S}}}\left(\frac{Y_{40 \mathrm{Ca}}}{Y_{32 \mathrm{~S}}}\right)^{1 / 2} \\
& \approx \exp \left(\frac{B_{28 \mathrm{Si}}-B_{32 \mathrm{~S}}-0.5\left(B_{32 \mathrm{~S}}-B_{40 \mathrm{Ca}}\right)}{k_{\mathrm{B}} T}\right)
\end{aligned}
$$




$$
=\exp \left(\frac{-1.25}{T_{9}}\right)
$$

where $T_{9}$ is the temperature in units of $10^{9} \mathrm{~K}$. Typical temperatures in the QNSE regions where the SiG elements are formed are $(3.4-4.0) \times 10^{9} \mathrm{~K}$ (a range of $15 \%$ in temperature); over this range $\Phi$ varies by $6 \%$, from 0.73 to 0.69 . Measuring $\Phi$ at a single epoch from the abundance ratios $Y_{28 \mathrm{Si}} / Y_{32 \mathrm{~S}}$ and $Y_{40 \mathrm{Ca}} / Y_{32 \mathrm{~S}}$ allows a test of whether the SiG material was produced in a QNSE state. With sufficient precision, measurement of $\Phi$ allows a determination of the temperature in the QNSE region before freeze-out. We assume for the remainder of this paper that such precision is available and that the QNSE temperature is a known quantity. Measuring $\Phi$ at multiple epochs when silicon features dominate the SNIa spectrum allows trends in the QNSE temperature to be assessed.

More generally, a double ratio of the form

$$
K=\frac{Y_{Z-2, A-4}}{Y_{Z, A}} \frac{Y_{Z^{\prime}+2, A^{\prime}+2}}{Y_{Z^{\prime}, A^{\prime}}} \approx \exp \left[\frac{\left(B_{Z-2, A-2}-B_{Z, A}\right)-\left(B_{Z^{\prime}, A^{\prime}}-B_{Z^{\prime}+2, A^{\prime}+4}\right)}{k_{\mathrm{B}} T}\right]
$$

is independent of $\rho, \theta, Y_{\mathrm{p}}$, and $Y_{\mathrm{n}}$. Additionally, if the isotopes in this ratio are major constituents of clusters in NQSE, then the argument of the exponential will be of order unity and $K$ will not vary strongly over the narrow range of temperature for which NQSE conditions attain. A relatively precise value of $K$ can then be specified from the ratio $\Phi$. Such a quasi-constant can also be defined for the FeG elements. Equations (6) and (7) imply that

$$
\Psi \equiv \frac{Y_{58 \mathrm{Ni}}}{Y_{54 \mathrm{Fe}}} \frac{Y_{28 \mathrm{Si}}}{Y_{32 \mathrm{~S}}} \approx \exp \left(\frac{6.36}{T_{9}}\right)
$$

where we again assume all ratios of nuclear partition functions are unity, and, by construction, $\Psi$ is independent of $\rho$ and $\theta$. Over the range of QNSE temperatures, $\Psi$ varies by $28 \%$ : from $\Psi=6.5$ at $T_{9}=3.4$ to $\Psi=4.9$ at $T_{9}=4.0$.

\subsection{A recipe to construct $Y_{\mathrm{e}}$ from the major elements}

For our simplified system consisting of the a few major SiG and FeG elements, Equation (2) may be written as

$$
Y_{\mathrm{e}}=Y_{28 \mathrm{Si}}\left[14+16 \frac{Y_{32 \mathrm{~S}}}{Y_{28 \mathrm{Si}}}+20 \frac{Y_{40 \mathrm{Ca}}}{Y_{32 \mathrm{~S}}} \frac{Y_{32 \mathrm{~S}}}{Y_{28 \mathrm{Si}}}+26 \frac{Y_{54 \mathrm{Fe}}}{Y_{28 \mathrm{Si}}}+28 \frac{Y_{58 \mathrm{Ni}}}{Y_{28 \mathrm{Si}}}+\frac{Y_{\mathrm{p}}}{Y_{28 \mathrm{Si}}}\right] .
$$

We factor out $Y_{28 \mathrm{Si}}$ because, as we show in $\$ 3$, the silicon yield is the least sensitive to changes in $\rho, T$, and $Y_{\mathrm{e}}$ in QNSE material. We may also drop $Y_{\mathrm{p}}$ since it much smaller $\left(Y_{\mathrm{p}}<10^{-4}\right)$ than the other abundances. 
The first step in reconstructing $Y_{\mathrm{e}}$ is to determine from observations the $Y_{28 \mathrm{Si}} / Y_{32 \mathrm{~S}}$ and $Y_{40 \mathrm{Ca}} / Y_{32 \mathrm{~S}}$ abundance ratios from strata with similar velocities. Measurement of these ratios determines the second and third terms of Equation (12). Their ratio also forms $\Phi$ (Equation (9)), which if near unity verifies that the SiG elements were synthesized in a QNSE environment. This relation may also be inverted to determine the temperature of the QNSE environment when the SiG elements were synthesized.

The next step is to measure the $Y_{54 \mathrm{Fe}} / Y_{28 \mathrm{Si}}$ abundance ratio. Usually it is difficult to extract the ${ }^{54} \mathrm{Fe}$ abundance from the iron lines. However, ${ }^{54} \mathrm{Fe}$ is the only iron isotope that is abundant in the regime where both ${ }^{28} \mathrm{Si}$ and ${ }^{32} \mathrm{~S}$ are also abundant, in the absence of significant mixing of the QNSE material with core material. The reason for this is that in NSE, where most of the mass is in the iron group, the requirement that $Z \approx A$ forces ${ }^{56} \mathrm{Ni}$ to be the dominant abundance. In contrast, for QNSE, most of the mass is in the Sigroup isotopes and this charge/mass constraint is lifted, so that the greater binding energy of the slightly neutron-rich ${ }^{54} \mathrm{Fe}$ results in ${ }^{54} \mathrm{Fe}$ having a larger abundance than ${ }^{56} \mathrm{Ni}$ ( $\mathrm{Hix}$ \& Thielemann 1996; Meyer et al. 1998; Iliadis 2007). In the absence of large-scale mixing the ${ }^{54} \mathrm{Fe}$ produced in the silicon-rich regions is physically separated from the ${ }^{56} \mathrm{Ni}$ produced deeper in the core, so that signatures of iron at early times from ${ }^{54} \mathrm{Fe}$ do not depend on the ${ }^{56} \mathrm{Ni}$ decay chain. Therefore, if iron features are detected in the early time spectra $(\approx 8 \mathrm{~d})$ at the same expansion velocities where $\mathrm{SiG}$ elements dominate the spectral features, they are produced by ${ }^{54} \mathrm{Fe}$. This result is due to material being in QNSE and is not dependent on any particular SNIa model.

The final step is to determine the $Y_{58 \mathrm{Ni}} / Y_{28 \mathrm{Si}}$ abundance ratio. Using Equation (11) we write

$$
\frac{Y_{58 \mathrm{Ni}}}{Y_{28 \mathrm{Si}}}=\frac{Y_{58 \mathrm{Ni}}}{Y_{54 \mathrm{Fe}}} \frac{Y_{54 \mathrm{Fe}}}{Y_{28 \mathrm{Si}}}=\Psi \frac{Y_{32 \mathrm{~S}}}{Y_{28 \mathrm{Si}}} \frac{Y_{54 \mathrm{Fe}}}{Y_{28 \mathrm{Si}}} .
$$

Thus, the last two terms of Equation $(12)$ are determined and may be rewritten as

$$
Y_{\mathrm{e}}=Y_{28 \mathrm{Si}}\left[14+16 \frac{Y_{32 \mathrm{~S}}}{Y_{28 \mathrm{Si}}}+20 \frac{Y_{40 \mathrm{Ca}}}{Y_{32 \mathrm{~S}}} \frac{Y_{32 \mathrm{~S}}}{Y_{28 \mathrm{Si}}}+26 \frac{Y_{54 \mathrm{Fe}}}{Y_{28 \mathrm{Si}}}+28 \Psi \frac{Y_{32 \mathrm{~S}}}{Y_{28 \mathrm{Si}}} \frac{Y_{54 \mathrm{Fe}}}{Y_{28 \mathrm{Si}}}\right] .
$$

Equation (14) is our principal result. Highly accurate measurement abundance determinations of the four quantities $Y_{28 \mathrm{Si}}, Y_{32 \mathrm{~S}} / Y_{28 \mathrm{Si}}, Y_{40 \mathrm{Ca}} / Y_{32 \mathrm{~S}}$, and $Y_{54 \mathrm{Fe}} / Y_{28 \mathrm{Si}}$ under the relevant temperatures is sufficient to determine $Y_{\mathrm{e}}$ to within $6 \%$ because these abundance dominate the QNSE composition. 


\subsection{Including non-major elements to refine the $Y_{\mathrm{e}}$ estimate}

The abundance of any $\alpha$-chain $\mathrm{SiG}$ element, which can be used to improve the accuracy of the $Y_{\mathrm{e}}$ determination, can be recovered using Equation (3) and $Y_{32 \mathrm{~S}} / Y_{28 \mathrm{Si}}$. For example, the abundance ratio $Y_{32 \mathrm{~S}} / Y_{36 \mathrm{Ar}}$ is related to $Y_{28 \mathrm{Si}} / Y_{32 \mathrm{~S}}$ by

$$
\frac{Y_{28 \mathrm{Si}}}{Y_{32 \mathrm{~S}}}=K_{2} \frac{Y_{32 \mathrm{~S}}}{Y_{36 \mathrm{Ar}}}
$$

with $K_{2} \approx \exp \left(-3.56 / T_{9}\right)$. As the temperature ranges from $T_{9}=3.4$ to $4.0, K_{2}$ varies from 0.35 to 0.41 , a variation of $16 \%$. Including $Y_{36 \mathrm{Ar}}$ in the sum for $Y_{\mathrm{e}}$ in Eq. (12), adds the term $36 K_{2}\left(Y_{32 \mathrm{~S}} / Y_{28 \mathrm{Si}}\right)^{2}$ to the right hand side of Eq. (14). Other $\alpha$-chain non-major elements may be added in a similar manner.

Deviation of $Y_{\mathrm{e}}$ from 0.5 is primarily due to the major element $Y_{54 \mathrm{Fe}}$ with contributions from other non-major $\mathrm{SiG}$ and FeG elements. For a more accurate determination of $Y_{\mathrm{e}}$, one can use the mass conservation, Equation (1), to determine the abundances of these nonmajor isotopes. For example, consider the case when $Y_{56 \mathrm{Ni}}$ and $Y_{30 \mathrm{Si}}$ are to be included. Treating $Y_{\mathrm{p}}$ and $Y_{\mathrm{n}}$ as trace abundance in the QNSE regions, Equation (1) becomes

$$
Y_{28 \mathrm{Si}}\left[28+32 \frac{Y_{32 \mathrm{~S}}}{Y_{28 \mathrm{Si}}}+40 \frac{Y_{40 \mathrm{Ca}}}{Y_{28 \mathrm{Si}}}+54 \frac{Y_{54 \mathrm{Fe}}}{Y_{28 \mathrm{Si}}}+58 \frac{Y_{58 \mathrm{Ni}}}{Y_{28 \mathrm{Si}}}\right]+Y_{56 \mathrm{Ni}}\left[56+30 \frac{Y_{30 \mathrm{Si}}}{Y_{56 \mathrm{Ni}}}\right]=1 \text {, }
$$

which we are going to solve for $Y_{56 \mathrm{Ni}}$ in the QNSE region. From Equation (3) the abundance ratio $Y_{30 \mathrm{Si}} / Y_{56 \mathrm{Ni}}$ may be written as

$$
\frac{Y_{30 \mathrm{Si}}}{Y_{56 \mathrm{Ni}}}=K_{3} \frac{Y_{28 \mathrm{Si}}}{Y_{56 \mathrm{Ni}}} \frac{Y_{58 \mathrm{Ni}}}{Y_{56 \mathrm{Ni}}}=K_{3}\left(\frac{Y_{28 \mathrm{Si}}}{Y_{56 \mathrm{Ni}}}\right)^{2} \frac{Y_{58 \mathrm{Ni}}}{Y_{28 \mathrm{Si}}},
$$

where $K_{3} \approx \exp \left(-39.3 / T_{9}\right)$. Substituting Equation (17) into Equation (16) and using previous relations gives

$$
\begin{aligned}
56 Y_{56 \mathrm{Ni}} & +30 K_{3} \frac{Y_{28 \mathrm{Si}}^{2}}{Y_{56 \mathrm{Ni}}}\left(\Psi \frac{Y_{32 \mathrm{~S}}}{Y_{28 \mathrm{Si}}} \frac{Y_{54 \mathrm{Fe}}}{Y_{28 \mathrm{Si}}}\right)=1-Y_{28 \mathrm{Si}} \times \\
& {\left[28+32 \frac{Y_{32 \mathrm{~S}}}{Y_{28 \mathrm{Si}}}+40 \frac{Y_{40 \mathrm{Ca}}}{Y_{32 \mathrm{~S}}} \frac{Y_{32 \mathrm{~S}}}{Y_{28 \mathrm{Si}}}+54 \frac{Y_{54 \mathrm{Fe}}}{Y_{28 \mathrm{Si}}}+58 \Psi \frac{Y_{32 \mathrm{~S}}}{Y_{28 \mathrm{Si}}} \frac{Y_{54 \mathrm{Fe}}}{Y_{28 \mathrm{Si}}}\right] . }
\end{aligned}
$$

Multiplying Equation (18) by $Y_{56 \mathrm{Ni}}$ thus yields a simple quadratic equation, $56 Y_{56 \mathrm{Ni}}^{2}-b Y_{56 \mathrm{Ni}}+$ $c=0$, with $c=30 K_{3} Y_{28 \mathrm{Si}}^{2} Y_{58 \mathrm{Ni}} / Y_{28 \mathrm{Si}}$ and $b$ being the right hand side of Equation (18). Taking $Y_{56 \mathrm{Ni}}$ as the positive root and substituting it into Equation 17 then gives $Y_{30 \mathrm{Si}}$. The derived $Y_{56 \mathrm{Ni}}$ and $Y_{30 \mathrm{Si}}$ abundances may then be used to refine the estimate for $Y_{\mathrm{e}}$ by adding the now 
known terms to Equation (14):

$$
\begin{aligned}
Y_{\mathrm{e}}= & Y_{28 \mathrm{Si}}\left[14+16 \frac{Y_{32 \mathrm{~S}}}{Y_{28 \mathrm{Si}}}+20 \frac{Y_{40 \mathrm{Ca}}}{Y_{32 \mathrm{~S}}} \frac{Y_{32 \mathrm{~S}}}{Y_{28 \mathrm{Si}}}+26 \frac{Y_{54 \mathrm{Fe}}}{Y_{28 \mathrm{Si}}}+28 \Psi \frac{Y_{32 \mathrm{~S}}}{Y_{28 \mathrm{Si}}} \frac{Y_{54 \mathrm{Fe}}}{Y_{28 \mathrm{Si}}}\right] \\
& +Y_{56 \mathrm{Ni}}\left[28+14 \frac{Y_{30 \mathrm{Si}}}{Y_{56 \mathrm{Ni}}}\right] .
\end{aligned}
$$

Abundances of other non-major elements may be added in a similar manner to the example given above to improve the determination of $Y_{\mathrm{e}}$.

\subsection{On the expected abundance trends with $Y_{\mathrm{e}}$}

In this section we section we seek a simple analytic relation between the abundances of the major QNSE elements, $Y_{28 \mathrm{Si}}, Y_{32 \mathrm{~S}}$ and $Y_{40 \mathrm{Ca}}$ with respect to $Y_{\mathrm{e}}$. We begin by re-writing Equation (3) as

$$
\frac{1}{f(\rho, T)} \frac{Y_{A Z}}{Y_{A^{\prime} Z^{\prime}}}=Y_{\mathrm{n}}^{A-A^{\prime}-\left(Z-Z^{\prime}\right)} Y_{\mathrm{p}}^{Z-Z^{\prime}}
$$

We may assume without loss of generality that $A>A^{\prime}$ and $Z>Z^{\prime}$. Now let

$$
w=\sum_{i \neq \text { protons }} Z_{i} Y_{A_{i} Z_{i}}
$$

which is identical to the definition of the electron fraction $Y_{\mathrm{e}}$ but without the free protons. We define, $v=Y_{A^{\prime} Z^{\prime}} f(\rho, T)$. Therefore, with this notation Eqn. 20 becomes,

$$
Y_{A_{i} Z_{i}}=v\left(1-Y_{\mathrm{e}}-w\right)^{A_{i}-A^{\prime}-\left(Z_{i}-Z^{\prime}\right)}\left(Y_{\mathrm{e}}-w\right)^{Z_{i}-Z^{\prime}} .
$$

Multiplying by $Z_{i}$ and summing,

$$
w=\sum_{i \neq \text { protons }} Z_{i} Y_{A_{i}, Z_{i}}=\sum_{i \neq \text { protons }} Z_{i} v_{i}\left(1-Y_{\mathrm{e}}-w\right)^{\left(A_{i}-A^{\prime}\right)-\left(Z_{i}-Z^{\prime}\right)}\left(Y_{\mathrm{e}}-w\right)^{Z_{i}-Z^{\prime}} .
$$

Since $Y_{\mathrm{e}} \rightarrow 0.5$, and $Y_{\mathrm{e}}<0.5$, therefore, $0<\left(1-Y_{\mathrm{e}}-w\right)<1$ and also that $0<$ $\left(Y_{\mathrm{e}}-w\right)<1$. This imples that the RHS of Eqn. 22 has most contribution from terms with smallest values of $\left(Z_{i}-Z^{\prime}\right)$ and $\left(A_{i}-A^{\prime}\right)-\left(Z_{i}-Z^{\prime}\right)$. Note that we have chosen $Z_{i}>Z^{\prime}$. For most major QNSE elements we may then choose $A^{\prime}=2 Z^{\prime}$ amd $A_{i}=2 Z_{i}$. For the $\mathrm{SiG}$ group pair ${ }^{28} \mathrm{Si}$ and ${ }^{32} \mathrm{~S}$, Equation 23 becomes

$$
w=v Z_{32 \mathrm{~S}}\left(1-Y_{\mathrm{e}}-w\right)^{2}\left(Y_{\mathrm{e}}-w\right)^{2},
$$


which is quartic in $w$. To order $Y_{\mathrm{e}}^{2}$, Equation (24) has the solution

$$
w=\frac{1}{2 v Z_{32 \mathrm{~S}}}\left[\left(2 Y_{\mathrm{e}}+1\right) \pm \sqrt{\left(4 Y_{\mathrm{e}} v Z_{32 \mathrm{~S}}+1\right)}\right]
$$

Substituting $Z_{32 \mathrm{~S}}=16$ and expanding the square root leads to a zeroth order term that is a constant and a first order term that is is linear in $Y_{\mathrm{e}}$. From the expression for $w$ in Eq. 23 , the largest contribution comes from $Z_{28 S i} Y_{28 \mathrm{Si}}$. We thus identify $Y_{28 \mathrm{Si}}$ as the constant term and the linear term in $Y_{\mathrm{e}}$ with $Y_{32 \mathrm{~S}}$. Finally, we identify $Y_{40 \mathrm{Ca}}$ with keeping the higher order terms in $Y_{\mathrm{e}}$ in Equation (24).

\section{Verification of QNSE from simulation models}

Here we suggest the trends predicted by our QNSE-based theoretical relations are manifested in the abundances derived from common 1D SNIa models. In the next subsection we describe the simulation model which we use to test our QNSE predictions.

\subsection{Description of simulation models}

We begin with the W7 model (Nomoto et al. 1984; Thielemann et al. 1986; Iwamoto et al. 1999) because the synthetic light cueves and spectra from W7 models have been extensively analyzed (e.g. Nugent et al. 1997; Hachinger et al. 2009; Jack et al. 2011; van Rossum 2012). W7 is a 1D explosion model with a parameterized flame speed that captures the stratified ejecta observed in Branch normal SNIa (Branch et al. 1993). For our purposes, a model is a series of temperature and density snapshots from ignition at time $=0 \mathrm{~s}$ to homologous expansion at time $=4.1 \mathrm{~s}$. The W7 model assumes a solar ${ }^{22} \mathrm{Ne}$ mass fraction uniformly distributed throughout the white dwarf. Our W7-like models change the assumed

value of the uniformly distributed ${ }^{22} \mathrm{Ne}$ mass fraction. Specifically, we control the value of the electron fraction $Y_{\mathrm{e}}$, which is set by the original composition and the pre-explosive convective simmering (Timmes et al. 2003; Piro \& Bildsten 2008; Chamulak et al. 2008; Townsley et al. 2009: Walker et al. 2012), by setting the mass fraction of ${ }^{22} \mathrm{Ne}: Y_{\mathrm{e}}=0.5-X\left({ }^{22} \mathrm{Ne}\right) / 22=$ $0.5-Q \cdot X\left({ }^{22} \mathrm{Ne}\right) \odot / 22$, where $Q$ is a multiplier on the solar ${ }^{22} \mathrm{Ne}$ mass fraction $X\left({ }^{22} \mathrm{Ne}\right)$.

The nucleosynthesis of these W7-like models (see Figure 1) is calculated by integrating a 489 isotope nuclear reaction network (Timmes 1999) over the thermodynamic trajectories of each Lagrangian mass shell. This post-processing calculation is not precisely self-consistent because the W7 thermodynamic trajectories have the built-in assumption of an energy release from the original W7 model, carbon+oxygen material complimented with a solar ${ }^{22} \mathrm{Ne}$ 
mass fraction. While changes to the abundance of ${ }^{22} \mathrm{Ne}$, hence $Y_{\mathrm{e}}$, slightly influence the energy generation rate (Hix \& Thielemann 1996, 1999), burned material still reaches QNSE conditions and our analysis should still hold.

\subsection{Verification of local QNSE relations}

First we test the validity of QNSE in the abundances synthesized in our W7-like SNIa models. We use local values of $\rho$ and T from these models to construct the QNSE predicted abundances.

Figure 2 shows the final mass fractions of the $\mathrm{SiG}$ elements ${ }^{28} \mathrm{Si},{ }^{32} \mathrm{~S}$, and ${ }^{40} \mathrm{Ca}$ between $0.8 M_{\odot}$ and $1.2 M_{\odot}$. Two cases for each element are shown, $Q=1.0$ and 2.0, representing 1.0 and 2.0 times the solar ${ }^{22} \mathrm{Ne}$ abundance, respectively. Solid lines represent the results from post-processed W7-like models and the symbols represent the results from our QNSE solutions. Figure 3 shows the final mass fractions of FeG elements ${ }^{54} \mathrm{Fe}$ and ${ }^{56} \mathrm{Ni}$ over the same mass range and white dwarf metallicities as in Figure 2. These figures show ${ }^{40} \mathrm{Ca}$ and ${ }^{54} \mathrm{Fe}$ have the largest systematic changes (up to a factor of two depending on the mass shell) within the silicon-rich region as the electron fraction varies. Also, a major conclusion from Figure 3 is that ${ }^{54} \mathrm{Fe}$ is the only iron isotope and the most abundant $\mathrm{FeG}$ element in the QNSE regime bound by regimes enclosing $0.8 M_{\odot}$ and $1.1 M_{\odot}$. This relation is also supported by Mazzali et al. (2013).

A weakly varying $\Phi$ (see Eq. (9)) implies $d \Phi / d T \simeq 0$. It follows that the constituent ratios $Y_{28 \mathrm{Si}} / Y_{32 \mathrm{~S}}$ and $Y_{32 \mathrm{~S}} / Y_{40 \mathrm{Ca}}$ reach an extremum at the same temperature. This property is evident in Figure 2 when there is a deflection in $Y_{28 \mathrm{Si}} / Y_{32 \mathrm{~S}}$ with mass coordinate there is a corresponding variation in $Y_{32 \mathrm{~S}} / Y_{40 \mathrm{Ca}}$. In addition, the individual abundances $Y_{28 \mathrm{Si}}, Y_{32 \mathrm{~S}}$, and $Y_{40 \mathrm{Ca}}$ reach an extremum at the same $(\rho, T)$ point where the ratios reach an extremum. In general, isotopes with $A=2 Z$ will have a QNSE abundance $Y_{\mathrm{AZ}}$ that scales as $\left(Y_{\mathrm{p}} Y_{\mathrm{n}}\right)^{n}$, where $n$ is a positive or negative integer. These isotopes can be expressed as a function of $Y_{28 \mathrm{Si}} / Y_{32 \mathrm{~S}}$ (Eq. (9) is one example) and will reach an extremum at the same $(\rho, T)$ point as $Y_{28 \mathrm{Si} \text {. The }}$ only assumption in deriving these properties is that the system achieves QNSE conditions, where most of the $\mathrm{SiG}$ elements are synthesized. The values of the ratios $Y_{28 \mathrm{Si}} / Y_{32 \mathrm{~S}}$ and $Y_{32 \mathrm{~S}} / Y_{40 \mathrm{Ca}}$ at the extremum will, of course, depend on the details of the explosion. Treating $\Psi$ (see Eq. (11)) as a quasi-constant and following arguments similar for the SiG elements, one finds $Y_{54 \mathrm{Fe}}, Y_{58 \mathrm{Ni}}$ and $Y_{28 \mathrm{Si}}$ also reach an extremum at the same $(\rho, T)$ point, as shown in Figure 3 . 


\subsection{Global abundances as predicted by QNSE}

Next we explore the global abundances of the $\mathrm{SiG}$ and FeG elements with $Y_{\mathrm{e}}$ when our W7-like models reach homologous expansion at $t=4.1 \mathrm{~s}$. Figure 4 shows the total ${ }^{28} \mathrm{Si},{ }^{32} \mathrm{~S}$, and ${ }^{40} \mathrm{Ca}$ molar abundances ejected as a function of the $Y_{\mathrm{e}}$. The curves correspond to postprocessing the W7-like thermodynamic trajectories and the symbols are the results from our analytical QNSE model. Both the post-processing and the model independent QNSE results suggest a nearly constant ${ }^{28} \mathrm{Si}$ yield with respect to $Y_{\mathrm{e}}$, a systematic quasi-linear ${ }^{32} \mathrm{~S}$ yield with respect to $Y_{\mathrm{e}}$, and a more complex trend for the global abundance of ${ }^{40} \mathrm{Ca}$ with $Y_{\mathrm{e}}$. Among the $\mathrm{SiG}$ elements, ${ }^{40} \mathrm{Ca}$ has the largest sensitivity to the electron fraction, in agreement with the trends seen in the local abundances. These results are in accord with trends explored in $\$ 2.4$.

\section{Possible application to observations}

That QNSE abundance ratios are manifest in our W7-like post-processing models suggests the QNSE relationships may be applicable to observations. We seek connections between abundances derived from a SNIa spectra that can be mapped to a $Y_{\mathrm{e}}$ of the silicongroup ejecta. The first step to such a mapping involves the most important test, whether

or not the derived abundances of major elements are in QNSE. In other words, are the abundances levels produced in QNSE retained duing the subsequent freeze-out?

\subsection{Verification of QNSE at freeze-out from simulation models}

Figure 11 shows the local abundances of the major $\mathrm{SiG}$ and FeG elements between mass shells $0.7 M_{\odot}$ and $1.3 M_{\odot}$ at $t=1.125 \mathrm{~s}$ (solid colored lines) and $t=4.10 \mathrm{~s}$ (dashed black lines) in one of our W7-like models. At $t=1.25 \mathrm{~s}$ the burning front has just passed over the $1.28 M_{\odot}$ mass coordinate and most of the synthesized ${ }^{28} \mathrm{Si},{ }^{32} \mathrm{~S}$, and ${ }^{40} \mathrm{Ca}$ have reached their equilibrium abundances. In this mass region, the peak temperatures, $(3-5) \times 10^{9} \mathrm{~K}$, and

peak densities, $(2-4) \times 10^{7} \mathrm{~g} \mathrm{~cm}^{-3}$, ensure QNSE conditions (Nomoto et al. 1984; Thielemann et al. 1986). The choice of $t=1.125 \mathrm{~s}$ is arbitrary and can be replaced by any epoch in any model when the material reaches QNSE conditions. At $t=4.10 \mathrm{~s}$ the explosion has entered homologous expansion and synthesis of all the elements has stopped due to the decreasing temperature. Complete freezeout does not occur for ${ }^{54} \mathrm{Fe}$ interior to $0.95 M_{\odot}$ at $t=1.25 \mathrm{~s}$ due to residual weak reactions. Figure 1 suggests that abundances generated when QNSE conditions apply are preserved during the subsequent freezeout. The abundance levels at this 
epoch may be reflected in the observed spectra over subsequent days. Therefore, applying the QNSE equations to recover $Y_{\mathrm{e}}$ from the major $\mathrm{SiG}$ and FeG elements as we have done in $\S 2$ is justified.

\subsection{Expected change in spectra due to change in $Y_{\mathrm{e}}$}

The next step to mapping the observed abundances into $Y_{\mathrm{e}}$ at explosion involves estimating the change in flux and luminosity as $Y_{\mathrm{e}}$ changes. Its important to estimate this change which dictates the level of resolution in $Y_{\mathrm{e}}$ mapping accessible from the observed abundances, if determined accurately from the spectra. There are several complicating factors that contribute to estimating acurate abundances from an observed spectra.

Below we estimate the change in flux with respect to $Y_{\mathrm{e}}$ using synthetic spectra from radiative transfer modeling.

We use the PHOENIX radiation transfer code (Hauschildt et al. 1997; Jack et al. 2009; Jack 2009; Jack et al. 2011) to produce synthetic spectra from our W7-like models. The thermodynamic profiles of the W7-like models end when the explosion reaches homologous expansion, about $4 \mathrm{~s}$ after ignition. The density, velocity, and abundance profiles are then homologously and adiabatically expanded to 5 days after the explosion using analytical expressions that account for the local decay of ${ }^{56} \mathrm{Ni}$ and ${ }^{56} \mathrm{Co}$. This is a reasonable assumption for SNIa after the initial break out (Arnett 1982). From day 5 onwards, we address LTE radiative transfer through the expanding remnant in 0.5 day increments to about 21 days to calculate synthetic spectra. At each of these 0.5 day increments, we solve an energy equation that includes the contribution of the adiabatic expansion, the energy deposition by $\gamma$-rays, and absorption and emission of radiation. As a result we always obtain a model atmosphere that is in radiative equilibrium.

Figure 5 shows the synthetic spectra at day 15, near peak luminosity, for the W7like models with $Q=0.0,1.0,2.0$, and 4.0, representing 0.0, 1.0, 2.0, and 4.0 times the

solar ${ }^{22} \mathrm{Ne}$ abundance, respectively. Changes in the synthetic spectra shown in Figure 5 are due to changes in the abundance of a given element. The SiG material has post-explosion homologous expansion velocities ranging between $9000-13000 \mathrm{~km} \mathrm{~s}^{-1}$ (e.g. Nugent et al. 1997; Hachinger et al. 2009; Jack et al. 2011; van Rossum 2012). The continuum optical depth of the SiG material is between 0.2-0.8, which was evaluated from the continuum opacity at $5500 \AA$ at peak luminosity. The wavelength resolution of the PHOENIX calculation is set to $1 \AA$ and the distance for the flux scale shown on the $y$-axis is the velocity of the outermost expanding shell times the time since the explosion. The PHOENIX model uses complete 
redistribution, with the same emission and absorption profile function with respect to the frequency spread around the center of the line frequency. Figure 5 suggests that the Ca II feature changes the most with $Y_{\mathrm{e}}$ when compared to S II and Si II features. The Si II line changes the least with variations in $Y_{\mathrm{e}}$. These trends, Ca being the most sensitive, $\mathrm{S}$ having a near linear dependence, and $\mathrm{Si}$ the least sensitive, are a reflection of the nucleosynthesis trends shown in Figure 4. We stress the LTE synthetic spectrum of our W7-like models is not compared to observational data, and thus our W7-like models may not accurately model real SNIa.

\section{Discussion}

We summarize our findings and conclusions of this work below.

1. We construct a mapping between $Y_{\mathrm{e}}$ at explosion and abundances of a few major elements from $\mathrm{SiG}$ and FeG. Specifically, we show that the abundance $Y_{28 \mathrm{Si}}$ and the abundance ratios $Y_{32 \mathrm{~S}} / Y_{28 \mathrm{Si}}, Y_{40 \mathrm{Ca}} / Y_{32 \mathrm{~S}}$, and $Y_{54 \mathrm{Fe}} / Y_{28 \mathrm{Si}}$ describe the complete basis to reconstruct the $Y_{\mathrm{e}}$ of silicon group material at explosion. This conclusion simply follows from the QNSE relations. If these four abundances are determined exactly, they are sufficient to recover $Y_{\mathrm{e}}$ to $6 \%$. This is because these isotopes dominate the composition of silicon-rich material and iron-rich material in QNSE.

2. From the widely successful Our W7-like simulations suggest that the major element abundances manifest QNSE trend at freeze-out. This might imply the abundances underlying an observed spectra obey the QNSE relations. This is one of our most important chief findings, which may allow one to a mapping of the major elements found determined from an observed spectra onto $Y_{\mathrm{e}}$ of the $\mathrm{SiG}$ material at explosion.

3. We find that among the major $\mathrm{SiG}$ elements, $\mathrm{Ca}$ abundance is expected to change the most with respect to $Y_{\mathrm{e}}$ at explosion. This fact follows from QNSE relations and manifest in the W7-like simulations as well. The Si abundance is not expected to change much as $Y_{\mathrm{e}}$ is varied. We predict that in the QNSE regime $Y_{54 \mathrm{Fe}}$ is the only isotope of iron and the only element in the FeG to have an abundance comparable with respect to the $\mathrm{SiG}$ elements. This conclusion also follow from QNSE equations.

4. Fig. 5 gives the reader a rough idea estimate of the change in spectral features with respect to $Y_{\mathrm{e}}$. These spectral features are driven by abundances that follow from QNSE. We conclude from the calculated synthetic spectra, A flux resolution of $0.1 \times 10^{15} \mathrm{erg}$ $\mathrm{cm}^{-2} \mathrm{~s}^{-1}$, if achieved, corresponds to a $\delta Y_{\mathrm{e}}=0.002$ resolution. This estimate is under 
the assumption that abundances can be determined precisely very accurately from an observed spectra and radiative transfer modeling. One of the avenues of recovering the abundance from a spectra is radiative transfer modeling of the spectra. Radiative transfer modeling treats the line formation in detail but is challenging and involves some modeling parameters. 
We thank Friedel Thielemann for providing the initial W7 thermodynamic trajectories. We also thank the anonymous reviewer for several suggestions which significantly improved the manuscript. This work was supported by the NSF through grant AST 0806720 (FXT) and PHY 08-022648 for the Frontier Center "Joint Institute for Nuclear Astrophysics" (JINA), by NASA through grant NNX09AD19G, and by the DOE through through grants DE-FG02-07ER41516, DE-FG02-08ER41570, DE-FG02-08ER41565 and DE-FG0287ER40317. SD received support from a SESE Postdoctoral fellowship at Arizona State University. 


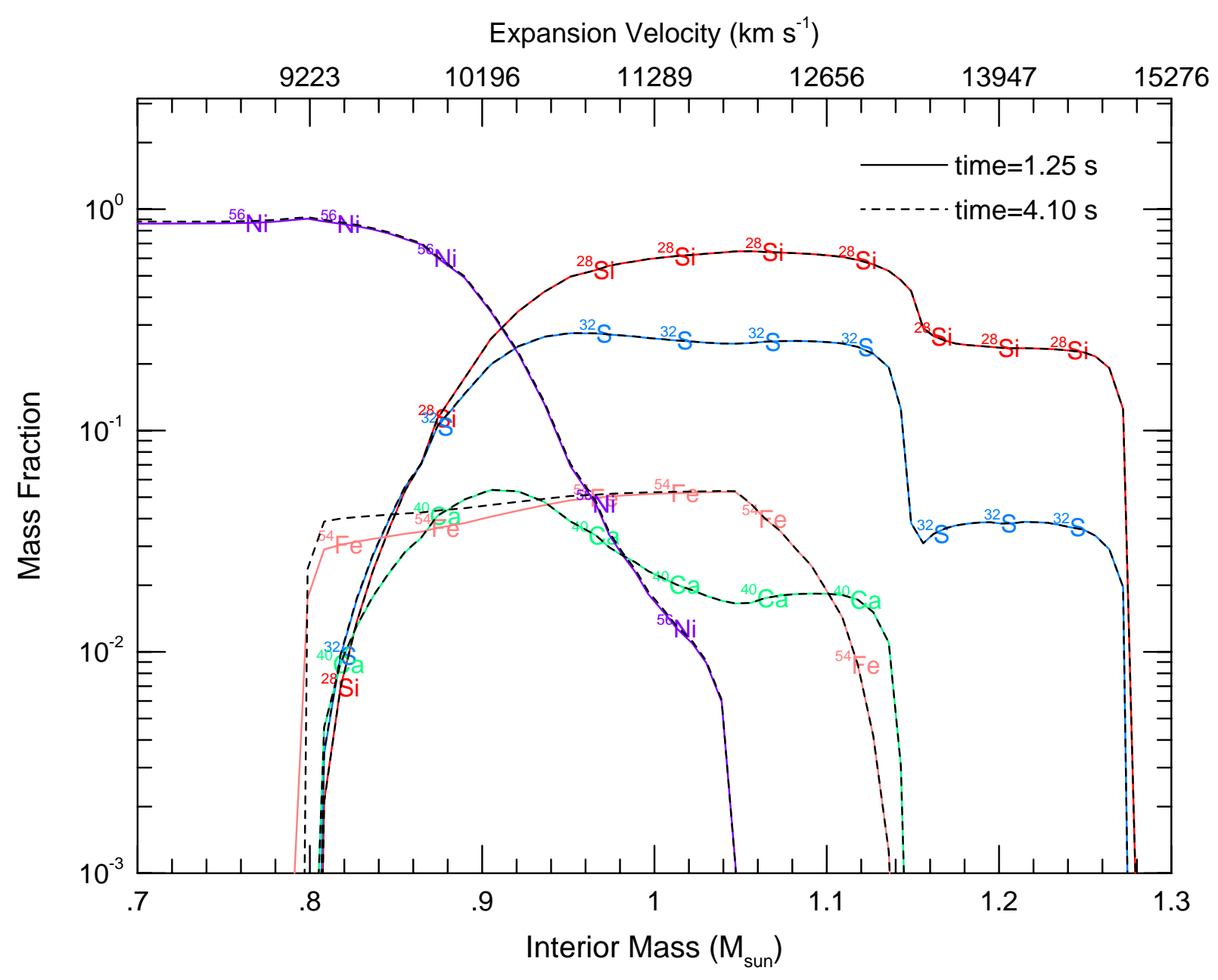

Fig. 1.- Mass fractions of the major elements during a W7-like explosion. Solid colored lines correspond to $t=1.25 \mathrm{~s}$ and and dashed black lines correspond to $t=4.10 \mathrm{~s}$ since ignition. The abundances produced by QNSE conditions at $t=1.25 \mathrm{~s}$ are the same as the final abundances when nuclear reactions freezeout at $t=4.0 \mathrm{~s}$. The expansion velocities on the upper $x$-axis are when the W7-like model reaches peak luminosity. 


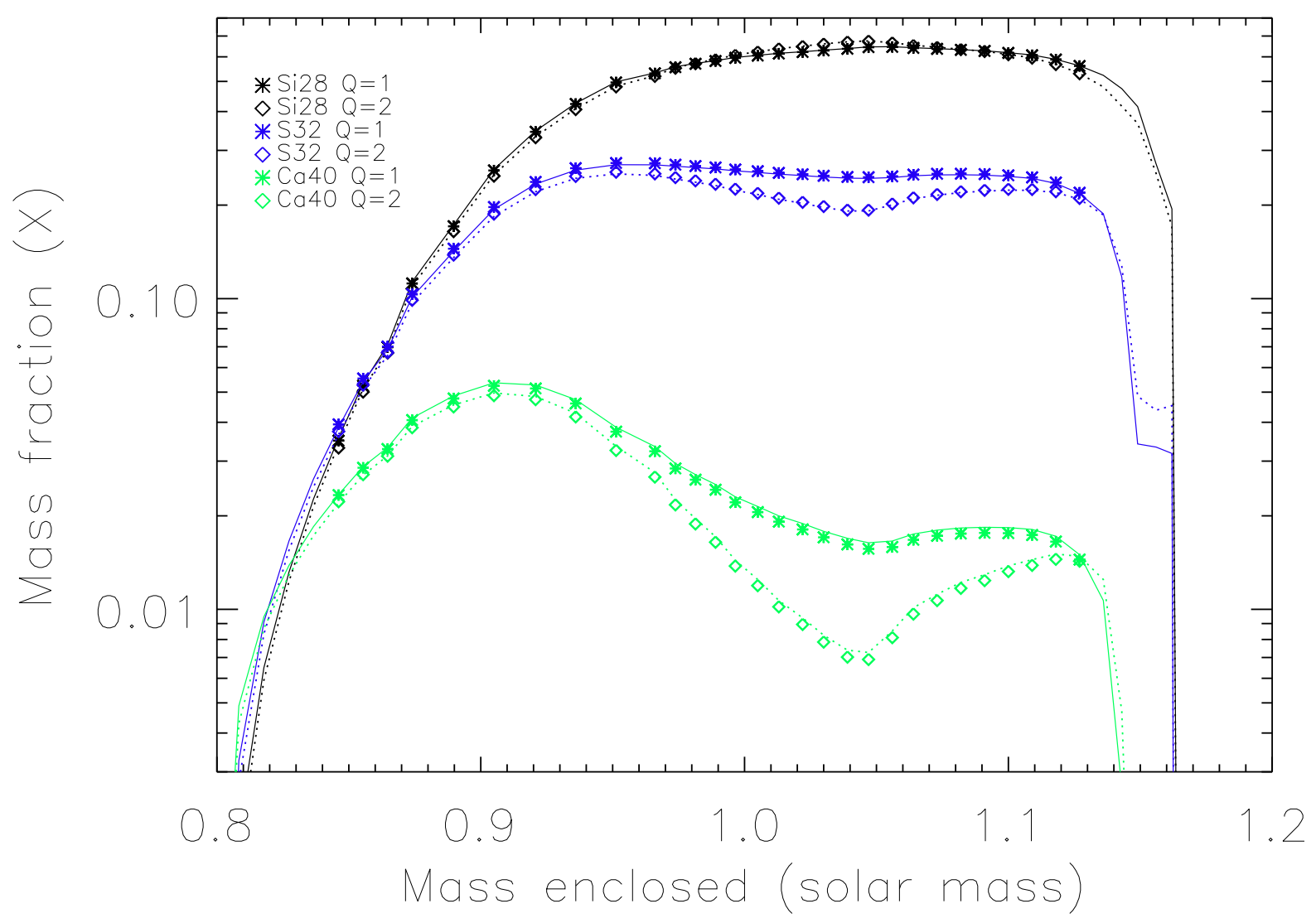

Fig. 2.- Mass fractions of the major SiG elements as a function of interior mass for the post-processed W7-like models (solid and dashed lines) and the QNSE calculations (symbols) at $t=1.19 \mathrm{~s}$. Two cases are shown, one for a solar ${ }^{22} \mathrm{Ne}$ abundance $(Q=1)$ and one a twice solar ${ }^{22} \mathrm{Ne}$ abundance $(Q=2)$. Overall, the agreement between the post-processed W7-like models and the analytical QNSE results are satisfactory. ${ }^{40} \mathrm{Ca}$ shows the largest change, up to a factor of two, while ${ }^{28} \mathrm{Si}$ is insensitive to changes in ${ }^{22} \mathrm{Ne}$. 


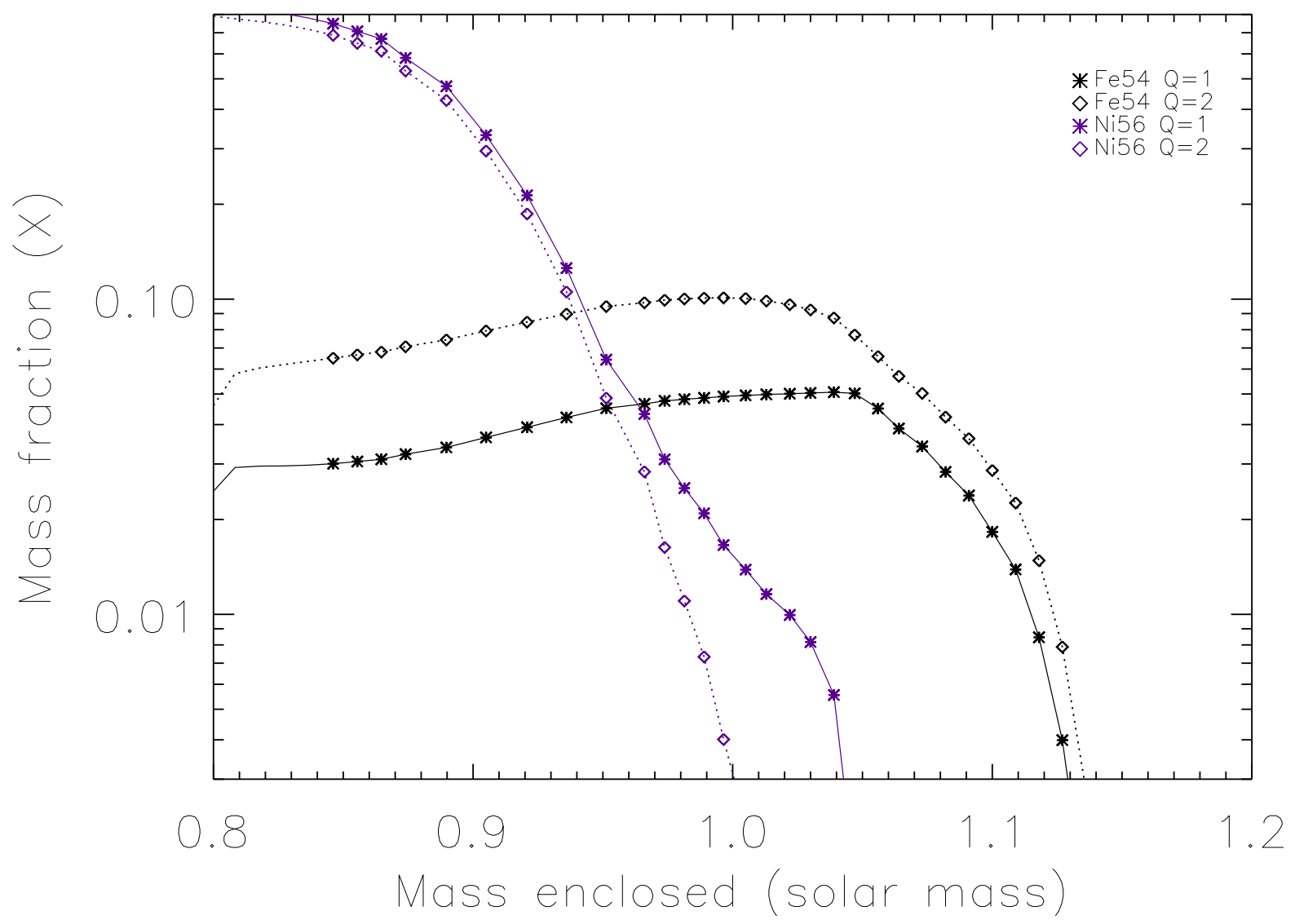

Fig. 3.- Same as Figure 2 but for the major FeG elements. ${ }^{54} \mathrm{Fe}$ shows the largest change with changes in ${ }^{22} \mathrm{Ne}$. Note that ${ }^{54} \mathrm{Fe}$ is the only significant iron isotope present when the SiG elements of Figure 2 are dominant. 


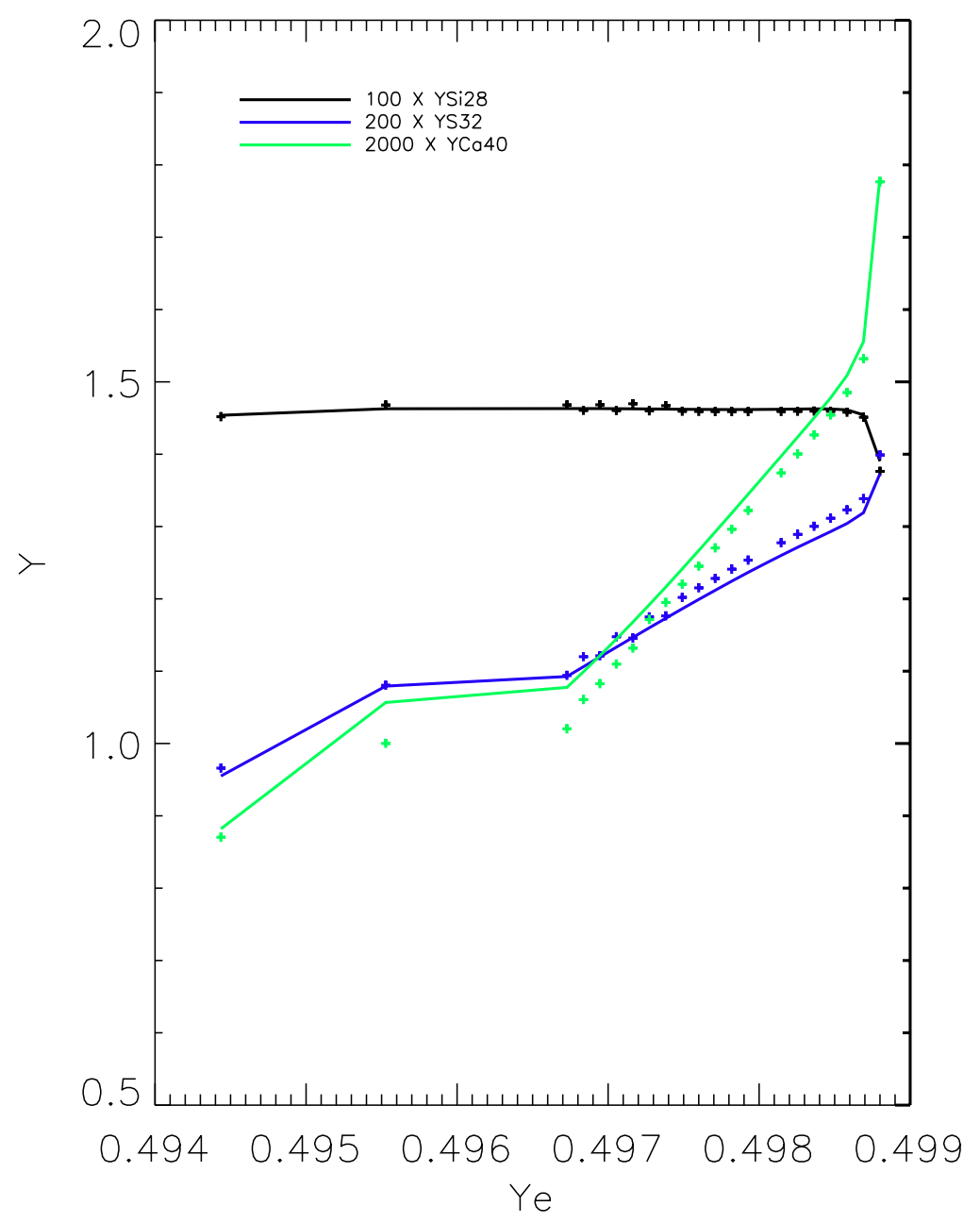

Fig. 4.- Global abundances of ${ }^{28} \mathrm{Si},{ }^{32} \mathrm{~S}$, and ${ }^{40} \mathrm{Ca}$ as a function of the electron fraction $Y_{\mathrm{e}}$ produced by the post-processed W7-like models (lines) and the analytical QNSE results (symbols). As for the trends in the local abundances (Figs. 1 3), ${ }^{28} \mathrm{Si}$ is independent of $Y_{\mathrm{e}}$, ${ }^{32} \mathrm{~S}$ shows a near linear dependence, and ${ }^{40} \mathrm{Ca}$ shows a more complex, but near quadratic, dependence with $Y_{\mathrm{e}}$. 


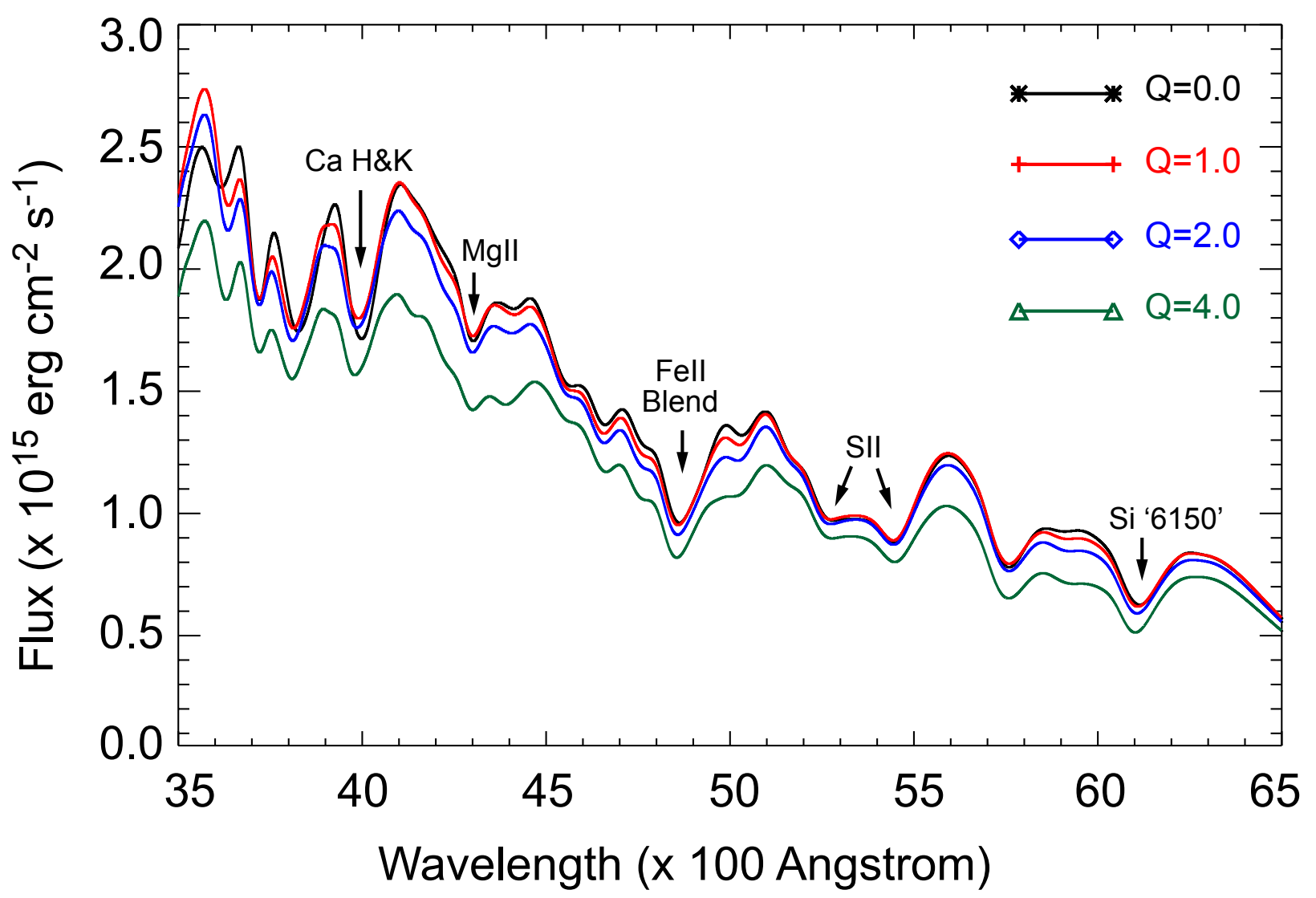

Fig. 5.- Synthetic spectra for the W7-like models with 0.0. 1.0, 2.0, and 4.0 times the solar ${ }^{22} \mathrm{Ne}$ abundance. Key abundance features are labeled. 


\section{REFERENCES}

Arnett, W. D. 1979, ApJ, 230, L37

—. 1982, ApJ, 253, 785

Arnett, W. D., Branch, D., \& Wheeler, J. C. 1985, Nature, 314, 337

Badenes, C., Bravo, E., \& Hughes, J. P. 2008, ApJ, 680, L33

Barbon, R., Benetti, S., Rosino, L., Cappellaro, E., \& Turatto, M. 1990, A\&A, 237, 79

Blondin, J. M., \& Mezzacappa, A. 2006, ApJ, 642, 401

Blondin, S., Mandel, K. S., \& Kirshner, R. P. 2011, A\&A, 526, A81+

Bodansky, D., Clayton, D. D., \& Fowler, W. A. 1968, ApJS, 16, 299

Bongard, S., Baron, E., Smadja, G., Branch, D., \& Hauschildt, P. H. 2006, ApJ, 647, 513

Branch, D., Dang, L. C., \& Baron, E. 2009, PASP, 121, 238

Branch, D., Fisher, A., \& Nugent, P. 1993, AJ, 106, 2383

Branch, D., Livio, M., Yungelson, L. R., Boffi, F. R., \& Baron, E. 1995, PASP, 107, 1019

Bravo, E., \& Badenes, C. 2011, MNRAS, 414, 1592

Bulbul, E., Smith, R. K., \& Loewenstein, M. 2012, ApJ, 753, 54

Chamulak, D. A., Brown, E. F., Timmes, F. X., \& Dupczak, K. 2008, ApJ, 677, 160

Chamulak, D. A., Meakin, C. A., Seitenzahl, I. R., \& Truran, J. W. 2012, ApJ, 744, 27

Chen, Y. Q., Nissen, P. E., Zhao, G., Zhang, H. W., \& Benoni, T. 2000, A\&AS, 141, 491

Colgate, S. A., Petschek, A. G., \& Kriese, J. T. 1980, ApJ, 237, L81

Conley, A., Guy, J., Sullivan, M., et al. 2011, ApJS, 192, 1

Domínguez, I., Höflich, P., \& Straniero, O. 2001, ApJ, 557, 279

Edvardsson, B., Andersen, J., Gustafsson, B., et al. 1993, A\&A, 275, 101

Ellis, R. S., Sullivan, M., Nugent, P. E., et al. 2008, ApJ, 674, 51

Feltzing, S., Holmberg, J., \& Hurley, J. R. 2001, A\&A, 377, 911 
Foley, R. J. 2012, ApJ, 748, 127

Foley, R. J., \& Kasen, D. 2011, ApJ, 729, 55

Foley, R. J., \& Kirshner, R. P. 2013, ArXiv e-prints

Foley, R. J., Sanders, N. E., \& Kirshner, R. P. 2011, ApJ, 742, 89

Gallagher, J. S., Garnavich, P. M., Berlind, P., et al. 2005, ApJ, 634, 210

Gallagher, J. S., Garnavich, P. M., Caldwell, N., et al. 2008, ApJ, 685, 752

Hachinger, S., Mazzali, P. A., Taubenberger, S., Pakmor, R., \& Hillebrandt, W. 2009, MNRAS, 399, 1238

Hauschildt, P. H., Baron, E., \& Allard, F. 1997, ApJ, 483, 390

Hicken, M., Wood-Vasey, W. M., Blondin, S., et al. 2009, ApJ, 700, 1097

Hix, W. R., \& Thielemann, F.-K. 1996, ApJ, 460, 869

—. 1999, ApJ, 511, 862

Höflich, P., Wheeler, J. C., \& Thielemann, F. K. 1998, ApJ, 495, 617

Howell, D. A. 2001, ApJ, 554, L193

-. 2011, Nature Communications, 2

Howell, D. A., Sullivan, M., Brown, E. F., et al. 2009, ApJ, 691, 661

Iliadis, C. 2007, Nuclear Physics of Stars (Wiley-VCH Verlag)

Iwamoto, K., Brachwitz, F., Nomoto, K., et al. 1999, ApJS, 125, 439

Jack, D. 2009, PhD thesis, Hamburg

Jack, D., Hauschildt, P. H., \& Baron, E. 2009, A\&A, 502, 1043

-. 2011, A\&A, 528, A141

Jackson, A. P., Calder, A. C., Townsley, D. M., et al. 2010, ApJ, 720, 99

Jha, S., Riess, A. G., \& Kirshner, R. P. 2007, ApJ, 659, 122

Kahabka, P., \& van den Heuvel, E. P. J. 1997, ARA\&A, 35, 69 
Kasen, D., Nugent, P., Thomas, R. C., \& Wang, L. 2004, ApJ, 610, 876

Kasen, D., Röpke, F. K., \& Woosley, S. E. 2009, Nature, 460, 869

Kasen, D., \& Woosley, S. E. 2007, ApJ, 656, 661

Kasen, D., Nugent, P., Wang, L., et al. 2003, ApJ, 593, 788

Kowalski, M., Rubin, D., Aldering, G., et al. 2008, ApJ, 686, 749

Krueger, B. K., Jackson, A. P., Calder, A. C., et al. 2012, ApJ, 757, 175

Krueger, B. K., Jackson, A. P., Townsley, D. M., et al. 2010, ApJ, 719, L5

Mannucci, F., Della Valle, M., \& Panagia, N. 2006, MNRAS, 370, 773

Matteucci, F., \& Greggio, L. 1986, A\&A, 154, 279

Mazzali, P., Sullivan, M., Hachinger, S., et al. 2013, ArXiv e-prints

Mazzali, P. A., Nomoto, K., Cappellaro, E., et al. 2001, ApJ, 547, 988

Mazzali, P. A., \& Podsiadlowski, P. 2006, MNRAS, 369, L19

Meng, X., \& Yang, W. 2010, ApJ, 710, 1310

Meyer, B. S., Krishnan, T. D., \& Clayton, D. D. 1998, ApJ, 498, 808

Neill, J. D., Sullivan, M., Howell, D. A., et al. 2009, ApJ, 707, 1449

Nomoto, K., Thielemann, F.-K., \& Yokoi, K. 1984, ApJ, 286, 644

Nordin, J., Östman, L., Goobar, A., et al. 2011, ApJ, 734, 42

Nugent, P., Baron, E., Branch, D., Fisher, A., \& Hauschildt, P. H. 1997, ApJ, 485, 812

Nugent, P., Phillips, M., Baron, E., Branch, D., \& Hauschildt, P. 1995, ApJ, 455, L147

Parthasarathy, M., Branch, D., Jeffery, D. J., \& Baron, E. 2007, New A Rev., 51, 524

Pedicelli, S., Bono, G., Lemasle, B., et al. 2009, A\&A, 504, 81

Perlmutter, S., Aldering, G., Goldhaber, G., et al. 1999, ApJ, 517, 565

Phillips, M. M. 1993, ApJ, 413, L105

Pinto, P. A., \& Eastman, R. G. 2000, ApJ, 530, 744 
Piro, A. L., \& Bildsten, L. 2008, ApJ, 673, 1009

Piro, A. L., \& Chang, P. 2008, ApJ, 678, 1158

Powell, L. C., Slyz, A., \& Devriendt, J. 2011, MNRAS, 414, 3671

Riess, A. G., Filippenko, A. V., Challis, P., et al. 1998, AJ, 116, 1009

Riess, A. G., Macri, L., Casertano, S., et al. 2011, ApJ, 730, 119

Rolleston, W. R. J., Smartt, S. J., Dufton, P. L., \& Ryans, R. S. I. 2000, A\&A, 363, 537

Röpke, F. K., Gieseler, M., Reinecke, M., Travaglio, C., \& Hillebrandt, W. 2006, A\&A, 453, 203

Scannapieco, E., \& Bildsten, L. 2005, ApJ, 629, L85

Silverman, J. M., Ganeshalingam, M., Li, W., \& Filippenko, A. V. 2012a, MNRAS, 425, 1889

Silverman, J. M., Kong, J. J., \& Filippenko, A. V. 2012b, MNRAS, 425, 1819

Silverman, J. M., Foley, R. J., Filippenko, A. V., et al. 2012c, ArXiv e-prints

Sullivan, M., Le Borgne, D., Pritchet, C. J., et al. 2006, ApJ, 648, 868

Sullivan, M., Conley, A., Howell, D. A., et al. 2010, MNRAS, 406, 782

Sullivan, M., Guy, J., Conley, A., et al. 2011, ApJ, 737, 102

Suzuki, N., Rubin, D., Lidman, C., et al. 2012, ApJ, 746, 85

Tang, S., \& Wang, Q. D. 2010, MNRAS, 408, 1011

Thielemann, F.-K., Nomoto, K., \& Yokoi, K. 1986, A\&A, 158, 17

Timmes, F. X. 1999, ApJS, 124, 241

Timmes, F. X., Brown, E. F., \& Truran, J. W. 2003, ApJ, 590, L83

Townsley, D. M., Jackson, A. P., Calder, A. C., et al. 2009, ApJ, 701, 1582

Travaglio, C., Hillebrandt, W., \& Reinecke, M. 2005, A\&A, 443, 1007

Twarog, B. A. 1980, ApJ, 242, 242

Umeda, H., Nomoto, K., Yamaoka, H., \& Wanajo, S. 1999, ApJ, 513, 861 
van den Heuvel, E. P. J., Bhattacharya, D., Nomoto, K., \& Rappaport, S. A. 1992, A\&A, 262,97

van Rossum, D. R. 2012, ArXiv e-prints

Walker, E. S., Hachinger, S., Mazzali, P. A., et al. 2012, MNRAS, 427, 103

Wang, B., \& Han, Z. 2012, New A Rev., 56, 122

Wang, B., Meng, X.-C., Wang, X.-F., \& Han, Z.-W. 2008, Chinese Journal of Astronomy and Astrophysics, 8, 71

Wang, C.-Y. 2011, MNRAS, 415, 83

Whelan, J., \& Iben, I. J. 1973, ApJ, 186, 1007

Wood-Vasey, W. M., Friedman, A. S., Bloom, J. S., et al. 2008, ApJ, 689, 377 